\title{
A GENERALIZATION OF A THEOREM ON CONTINUOUS SELECTIONS
}

\author{
E. MICHAEL
}

(Communicated by Dennis Burke)

\begin{abstract}
The author's selection theorem for functions with finite-dimensional domain is sharpened in two directions.
\end{abstract}

\section{INTRODUCTION}

The purpose of this note is to sharpen the following theorem in two related directions, and to give some applications. The relevant definitions, which have all appeared in the literature, will be reviewed later in this introduction.

Theorem 1.1 [3, Theorem 1.2]. Let $X$ be paracompact, $Y$ completely metrizable, and $\varphi: X \rightarrow 2^{Y}$ l.s.c. with $\varphi(x) \in \mathscr{F}(Y)$ for all $x \in X$. Suppose that $A \subset X$ is closed, that $\operatorname{dim}_{X} X \backslash A \leq n+1$, and that $\{\varphi(x): x \in X\}$ is equi-LC $C^{n}$. Then every selection $g$ for $\varphi \mid A$ extends to a selection $f$ for $\varphi \mid U$ for some open $U \supset A$, and one can take $U=X$ if $\varphi(x)$ is $C^{n}$ for all $x \in X \backslash A$.

We now introduce some additional terminology. Suppose that $\varphi: X \rightarrow$ $\mathscr{P}(Y)$, where $\mathscr{P}(Y)=\{E: E \subset Y\}$. We define $\tilde{\varphi}: X \rightarrow \mathscr{P}(X \times Y)$ by $\tilde{\varphi}(x)=\{x\} \times \varphi(x)$, and (following [3, p. 574]) we say that the function $\varphi$ is equi- $L C^{n}$ if $\{\tilde{\varphi}(x): x \in X\}$ is an equi- $L C^{n}$ collection of subsets of $X \times Y$. This property of $\varphi$ is strictly weaker than $\{\varphi(x): x \in X\}$ being an equi- $L C^{n}$ collection of subsets of $Y$ (see Corollary 1.5), but it suffices for Theorem 1.1 (see Theorem 1.2) and it is conserved under a natural and useful operation which does not conserve $\{\varphi(x): x \in X\}$ being equi- $L C^{n}$ (see Lemma 5.1).

The following result generalizes Theorem 1.1. Part (a) of this generalization answers a question which was raised by the author on p. 575 of [3], and which had originated in a conversation between the author and S. Eilenberg.

Theorem 1.2. Theorem 1.1 can be sharpened simultaneously in two directions:

(a) The assumption that $\{\varphi(x): x \in X\}$ is equi- $L C^{n}$ can be weakened to assuming only that $\varphi$ is equi- $L C^{n}$.

Received by the editors October 8, 1987 and, in revised form, February 16, 1988 and May 12, 1988.

1980 Mathematics Subject Classification (1985 Revision). Primary 54C65, 54C99.

Key words and phrases. Continuous selections, equi- $L C^{n}, G_{\delta}$-set. 
(b) The assumption that $\varphi(x) \in \mathscr{F}(Y)$ for every $x \in X$ can be weakened to assuming only that there exists $a G_{\delta}$-subset $D$ of $X \times Y$ such that $\tilde{\varphi}(x) \in \mathscr{F}(D)$ for every $x \in X$.

As we shall see in $\S 2$, Theorem 1.2 follows almost immediately from Theorem 1.1 in the special case of metrizable $X$; the difficulty consists in proving it for all paracompact $X$, and thus obtaining a genuine generalization of Theorem 1.1. This is an unusual situation, since paracompact spaces are the natural domains for almost all selection theorems and, as a rule, it is no easier to prove a selection theorem (such as Theorem 1.1) for metrizable $X$ than for paracompact $X$. Theorem 1.2 presents a striking exception to this rule. The only other such exception known to the author is [4, Theorem 1.2], whose proof required the development of a special technique which was of some independent interest. The proof of Theorem 1.2 also depends on a-quite different-special technique, and it is this technique, as much as the theorem itself and its applications, which gives Theorem 1.2 its significance.

It is not easy to further weaken the conditions in Theorem 1.2. In particular, (a) cannot be weakened to simply assume that every $\varphi(x)$ is $L C^{n}$, even when $n=0$ (see [5, Example 10.6]), and (b) cannot be weakened to simply assume that every $\varphi(x)$ is a $G_{\delta}$ in $Y$, even when $n=-1$ (see [2, Example 6.1]).

The following result, which is applied in $\S 6$, is proved with the aid of both parts of Theorem 1.2. We adopt the convention that, if $D \subset X \times Y$ and $x \in X$, then $D(x)=\{y \in Y:(x, y) \in D\}$; moreover, if $\varphi: X \rightarrow \mathscr{P}(Y)$, then $\varphi_{D}: X \rightarrow \mathscr{P}(Y)$ is defined by $\varphi_{D}(x)=\varphi(x) \cap D(x)$.

Corollary 1.3. Let $X$ be paracompact with $\operatorname{dim} X \leq n+1, Y$ completely metrizable, and $\varphi: X \rightarrow \mathscr{F}(Y)$ l.s.c. and equi-LC ${ }^{n}$. Suppose that $D \subset X \times Y$ is open and that $\varphi_{D}(x)$ is nonempty and $C^{n}$ for all $x \in X$. Then $\varphi_{D}$ has a selection.

Let us now briefly define the concepts in Theorem 1.1. A space $Y$ is completely metrizable if it can be metrized with a complete metric. Letting $\mathscr{P}(Y)=$ $\{E: E \subset Y\}$, we define $2^{Y}=\{E \in \mathscr{P}(Y): E \neq \varnothing\}$ and $\mathscr{F}(Y)=\left\{E \in 2^{Y}: E\right.$ closed in $Y\}$. A function $\varphi: X \rightarrow 2^{Y}$ is l.s.c. if $\{x \in X: \varphi(x) \cap V \neq \varnothing\}$ is open in $X$ for every open $V$ in $Y$. A selection for $\varphi: X \rightarrow 2^{Y}$ is a continuous $f: X \rightarrow Y$ such that $f(x) \in \varphi(x)$ for every $x \in X$. The inequality $\operatorname{dim}_{X} X \backslash A \leq n+1$ means that $\operatorname{dim} B \leq n+1$ for every closed (in $X$ ) set $B \subset X \backslash A$.

To define $C^{n}$ and equi- $L C^{n}$, we introduce the following notation, which will be used throughout this paper: If $F \subset E$, then $F \prec E$ will mean that, for all $m \leq n$, every continuous $g: S^{m} \rightarrow F$ extends to a continuous $f: B^{m+1} \rightarrow E$ (where $S^{m}$ is the $m$-sphere and $B^{m+1}$ the $(m+1)$-ball). Now $E$ is called $C^{n}$ if $E \prec E$, and $\mathscr{E} \subset 2^{Y}$ is called equi- $L C^{n}$ if, for every $y \in \bigcup \mathscr{E}$, every neighborhood $W$ of $y$ in $Y$ contains a neighborhood $V$ of $y$ in $Y$ such that $V \cap E \prec W \cap E$ for every $E \in \mathscr{E}$. 
We now pause for a simple characterization of equi- $L C^{n}$ functions $\varphi: X \rightarrow$ $\mathscr{P}(Y)$, as defined after Theorem 1.1.

Lemma 1.4. If $\varphi: X \rightarrow \mathscr{P}(Y)$, then the following are equivalent.

(a) $\varphi$ is equi- $L C^{n}$.

(b) If $x^{*} \in X, y^{*} \in \varphi(x)$, and if $W$ is a neighborhood of $y^{*}$ in $Y$, then $\left(x^{*}, y^{*}\right)$ has a neighborhood $U \times V$ in $X \times Y$ such that $V \cap \varphi(x) \prec$ $W \cap \varphi(x)$ for every $x \in U$.

In contrast to Lemma $1.4,\{\varphi(x): x \in X\}$ is equi- $L C^{n}$ if and only if 1.4(b) is satisfied with $U=X$. We thus obtain the following corollary.

Corollary 1.5. If $\varphi: X \rightarrow \mathscr{P}(Y)$, and if $\{\varphi(x): x \in X\}$ is equi- $L C^{n}$, then $\varphi$ is equi- $L C^{n}$. $^{1}$

The easy proof of Theorem 1.2 for metrizable $X$ is given in $\S 2$. The technique needed to prove Theorem 1.2 in full generality is developed in $\S 3$, and Theorem 1.2 is then proved in $\S 4$. Corollary 1.3 is proved in $\S 5$, and some applications are obtained in $\S 6$. Finally, $\S 7$ is devoted to examples.

\section{Proof of Theorem 1.2 for Metrizable $X$}

Embed $X$ in a completely metrizable space $X^{\prime}$, and let $D^{\prime}$ be a $G_{\delta}$-subset of $X^{\prime} \times Y$ such that $D^{\prime} \cap(X \times Y)=D$. Since $X^{\prime}$ and $Y$ are completely metrizable, so is $X^{\prime} \times Y$ and hence so is $D^{\prime}$. Also $\tilde{\varphi}(x) \in \mathscr{F}\left(D^{\prime}\right)$ for every $x \in X$. Hence $\tilde{\varphi}: X \rightarrow \mathscr{F}\left(D^{\prime}\right)$ satisfies all the hypotheses of Theorem 1.1 (with $Y$ replaced by $D^{\prime}$ and $\varphi$ by $\left.\tilde{\varphi}\right)$.

Now let $g$ be a selection for $\varphi \mid A$. Define $\tilde{g}: X \rightarrow D^{\prime}$ by $\tilde{g}(x)=(x, g(x))$. Then $\tilde{g}$ is a selection for $\tilde{\varphi} \mid A$, so, by Theorem 1.1 applied to $\tilde{\varphi}$, this $\tilde{g}$ extends to a selection $\tilde{f}$ for $\tilde{\varphi} \mid U$ for some open $U \supset A$; moreover, if $\varphi(x)$ is $C^{n}$ for every $x \in X \backslash A$, then $\tilde{\varphi}(x)$ (which is homeomorphic to $\varphi(x)$ ) is also $C^{n}$ for $x \in X \backslash A$, so one can take $U=X$. Now define $f: X \rightarrow Y$ by $f=\pi_{2} \circ \tilde{f}$, where $\pi_{2}: X \times Y \rightarrow Y$ is the projection. This $f$ is the required extension of $g$.

It should be remarked that the validity of part (a) of Theorem 1.2 for metrizable $X$ was already established in [3, Theorem 9.2].

\section{SOME LEMMAS}

Throughout this section, $X$ is a topological space and $(Y, d)$ a metric space. If $\varphi: X \rightarrow 2^{Y}$, then $\operatorname{Gr} \varphi$ (the graph of $\varphi$ ) denotes $\{(x, y) \in X \times Y: y \in$ $\varphi(x)\}$. The half-open interval $(0,1]$ will be denoted by $J$.

Lemma 3.1. Suppose $\mathscr{U}$ is a collection of open rectangles $U=U^{\prime} \times U^{\prime \prime}$ in $X \times Y$. Define $u: \cup \mathscr{U} \rightarrow J$ by

$$
u(x, y)=\sup \left\{r \in J:\{x\} \times B_{r}(y) \subset U \text { for some } U \in \mathscr{U}\right\},
$$

\footnotetext{
${ }^{1}$ The converse is false; see Example 7.1.
} 
and define $\psi: \cup \mathscr{U} \rightarrow \mathscr{F}(J)$ by $\psi(x, y)=(0, u(x, y)]$. Then:

(a) $\psi$ is l.s.c.

(b) If $(x, y),\left(x, y^{\prime}\right) \in \bigcup \mathscr{U}$, then $\left|u(x, y)-u\left(x, y^{\prime}\right)\right| \leq d\left(y, y^{\prime}\right)$.

(c) If $(x, y) \in \bigcup \mathscr{U}$ and $\left(x, y^{\prime}\right) \notin \bigcup \mathscr{U}$, then $u(x, y) \leq d\left(y, y^{\prime}\right)$.

Proof. Routine verification.

Lemma 3.2. Let $\varphi: X \rightarrow 2^{Y}$ be equi- $L C^{n}$. Then for each $i \in \omega$ there exists a collection $\mathscr{U}_{i}$ of open rectangles $U=U^{\prime} \times U^{\prime \prime}$ in $X \times Y$ covering $\operatorname{Gr} \varphi$ such that, if $u_{i}: \cup \mathscr{U}_{i} \rightarrow J$ is as in Lemma 3.1, then

$$
B_{r}(y) \cap \varphi(x) \prec B_{1 / i}(y) \cap \varphi(x)
$$

whenever $(x, y) \in \bigcup \mathscr{U}_{i}$ and $r<u_{i}(x, y)$.

Proof. Fix $i \in \omega$. By Lemma 1.4, each $\left(x^{*}, y^{*}\right) \in \operatorname{Gr} \varphi$ has a neighborhood $U=U^{\prime} \times U^{\prime \prime}$ in $X \times Y$ such that $U^{\prime \prime} \cap \varphi(x) \prec B_{1 / 2 i}\left(y^{*}\right) \cap \varphi(x)$ for every $x \in U^{\prime}$. We may suppose that $\operatorname{diam} U^{\prime \prime}<1 / 2 i$, so that $U^{\prime \prime} \cap \varphi(x) \prec B_{1 / i}(y) \cap \varphi(x)$ whenever $(x, y) \in U$. Letting $\mathscr{U}_{i}$ be the collection of all such $U$, it follows from the definition of $u_{i}$ that $\mathscr{U}_{i}$ has the required property.

Lemma 3.3. Let $\varphi: X \rightarrow 2^{Y}$. For each $i \in \omega$, let $\mathscr{U}_{i}$ be a collection of open rectangles in $X \times Y$ covering $\operatorname{Gr} \varphi$, and let $u_{i}: \cup \mathscr{U}_{i} \rightarrow J$ and $\psi_{i}: \cup \mathscr{U}_{i} \rightarrow$ $\mathscr{F}(J)$ be as in Lemma 3.1. Let $E=\bigcap_{i}\left(\cup \mathscr{U}_{i}\right)$, let $Z=J^{\omega}$, define $\psi: E \rightarrow$ $\mathscr{F}(Z)$ by $\psi(x, y)=\prod_{i} \psi_{i}(x, y)$, and define $\theta: X \rightarrow 2^{Y \times Z}$ by

$$
\theta(x)=\{(y, z) \in Y \times Z: y \in \varphi(x), z \in \psi(x, y)\} .
$$

Then:

(a) $\psi$ is l.s.c.

(b) If $\varphi$ is l.s.c., so is $\theta$.

(c) If $x \in X$, and if $\varphi(x) \in \mathscr{F}(E(x))$, then $\theta(x) \in \mathscr{F}(Y \times Z)$.

(d) $\theta(x)$ is homeomorphic to $\varphi(x) \times Z$ for all $x \in X$.

(e) If $x \in X$, and if $\varphi(x)$ is $C^{n}$, then $\theta(x)$ is $C^{n}$.

(f) If $\varphi$ is equi- $L C^{n}$, and if the collections $\mathscr{U}_{i}$ are chosen as in Lemma 3.2 , then $\{\theta(x): x \in X\}$ is equi- $L C^{n}$.

(g) If $A \subset X$ is paracompact ${ }^{2}$, then for every selection $g$ for $\varphi \mid A$ there exists a selection $g^{\prime}$ for $\theta \mid A$ such that $\pi_{1} \circ g^{\prime}=g$ (where $\pi_{1}: Y \times Z \rightarrow Y$ is the projection).

Proof.

(a) Each $\psi_{i}$ is l.s.c. by $3.1(a)$, so $\psi$ is also 1.s.c.

(b) From (a) and the definition of $\theta$.

(c) Suppose $(y(j), z(j)) \rightarrow(y, z)$ in $Y \times Z$, with $(y(j), z(j)) \in \theta(x)$ for all $j$, and let us show that $(y, z) \in \theta(x)$.

\footnotetext{
${ }^{2}$ It suffices if $A$ is normal and countably paracompact.
} 
First, let us check that $y \in \varphi(x)$. Suppose not. Then $y \notin E(x)$, because $y(j) \in \varphi(x)$ and $\varphi(x)$ is relatively closed in $E(x)$. Hence $(x, y) \notin \bigcup \mathscr{U}_{i}$ for some $i$, so $u_{i}(x, y(j)) \rightarrow 0$ for this $i$ by $3.1(\mathrm{c})$; hence $z_{i}(j) \rightarrow 0$ (because $\left.z_{i}(j) \in \psi_{i}(x, y(j))\right)$, and thus $z_{i}(j) \nrightarrow z_{i}$ (because $z_{i} \neq 0$ since $z \in Z$ ), which is impossible because $z(j) \rightarrow z$.

Next, let us show that $z \in \psi(x, y)$. For all $i$ we have $z_{i}(j) \leq u_{i}(x, y(j)) \rightarrow$ $u_{i}(x, y)$ (by 3.1(b)) and $z_{i}(j) \rightarrow z_{i}$, so $z_{i} \leq u_{i}(x, y)$ and thus $z_{i} \in \psi_{i}(x, y)$. Hence $z \in \psi(x, y)$.

(d) This follows from the fact that, by 3.1(b), the restriction $u_{i} \mid(\{x\} \times \varphi(x))$ is continuous for all $x \in X$ and all $i$.

(e) This follows from (d), since the product of two $C^{n}$ spaces is $C^{n}$.

(f) Let $\left(y^{*}, z^{*}\right) \in Y \times Z$, and let $W \times L$ be a neighborhood of $\left(y^{*}, z^{*}\right)$ in $Y \times Z$. We will show that there is a neighborhood $V \times H$ of $\left(y^{*}, z^{*}\right)$ in $Y \times Z$ such that $(V \times H) \cap \theta(x) \prec(W \times L) \cap \theta(x)$ for all $x \in X .^{3}$

For $\delta>0$, define $N_{\delta}\left(z^{*}\right)=\left\{z \in Z:\left|z_{i}-z_{i}^{*}\right|<\delta\right.$ for $\left.i \leq 1 / \delta\right\}$. Pick $\gamma>0$ such that $N_{3 \gamma}\left(z^{*}\right) \subset L$. We may assume that $L=N_{3 \gamma}\left(z^{*}\right)$ and that $W \subset B_{y}\left(y^{*}\right)$.

Let us begin by showing that, if $x \in X$, if $y, y^{\prime} \in W \cap \varphi(x)$, and if $N_{\gamma}\left(z^{*}\right) \cap$ $\psi(x, y) \neq \varnothing$, then $L \cap \psi\left(x, y^{\prime}\right) \neq \varnothing$. Indeed, pick $z \in N_{\gamma}\left(z^{*}\right) \cap \psi(x, y)$. Now $y, y^{\prime} \in W \subset B_{\gamma}\left(y^{*}\right)$, so $d\left(y, y^{\prime}\right)<2 \gamma$ and hence $\left|u_{i}(x, y)-u_{i}\left(x, y^{\prime}\right)\right|<2 \gamma$ for all $i$ by $3.1(\mathrm{~b})$. Thus there is a $z^{\prime} \in \psi\left(x, y^{\prime}\right)$ such that $\left|z_{i}^{\prime}-z_{i}\right|<2 \gamma$ for all $i$. Now $\left|z_{i}^{\prime}-z_{i}^{*}\right|<2 \gamma+\gamma=3 \gamma$ for $i \leq 1 / 3 \gamma$, so $z^{\prime} \in N_{3 \gamma}\left(z^{*}\right) \subset L$. Hence $z^{\prime} \in L \cap \psi\left(x, y^{\prime}\right)$.

Next, let us show that there is a neighborhood $V \times K$ of $\left(y^{*}, z^{*}\right)$ in $Y \times Z$ such that $V \subset W$ and, if $x \in X$ and $(V \times K) \cap \theta(x) \neq \varnothing$, then $V \cap \varphi(x) \prec W \cap$ $\varphi(x)$. Indeed, pick $j \in \omega$ such that $B_{2 / j}\left(y^{*}\right) \subset W$, and let $\varepsilon=\min \left(\frac{1}{3} z_{j}^{*}, \frac{1}{j}\right)$. Let $V=B_{\varepsilon}\left(y^{*}\right)$, and let $K=\left\{z \in Z: z_{j}>2 \varepsilon\right\}$. To sees that this works, suppose $x \in X$ and $(V \times K) \cap \theta(x) \neq \varnothing$, and pick $(y, z) \in(V \times K) \cap \theta(x)$. Then $y \in \varphi(x), d\left(y, y^{*}\right)<\varepsilon$, and $2 \varepsilon<z_{j} \leq u_{j}(x, y)$, so, by the property of $\mathscr{U}_{j}$ in Lemma 3.2,

$$
V \cap \varphi(x) \subset B_{2 \varepsilon}(y) \cap \varphi(x) \prec B_{1 / j}(y) \cap \varphi(x) \subset B_{2 / j}\left(y^{*}\right) \cap \varphi(x) \subset W \cap \varphi(x),
$$

and hence $V \cap \varphi(x) \prec W \cap \varphi(x)$.

Now let $V$ and $K$ be as in the previous paragraph, and define $H=K \cap$ $N_{y}\left(z^{*}\right)$. That completes the construction of $V$ and $H$, and it remains to verify that $(V \times H) \cap \theta(x) \prec(W \times L) \cap \theta(x)$ for all $x \in X$. Since that is clear if $(V \times H) \cap \theta(x)=\varnothing$, we may suppose that $(V \times H) \cap \theta(x) \neq \varnothing$. We must show that, for $m \leq n$, every continuous $g: S^{m} \rightarrow(V \times H) \cap \theta(x)$ extends to a continuous $f: B^{m+1} \rightarrow(W \times L) \cap \theta(x)$. In the following construction of $f$, we let $\pi_{1}: Y \times Z \rightarrow Y$ and $\pi_{2}: Y \times Z \rightarrow Z$ denote the projections.

${ }^{3}$ Note that this will be shown for all $\left(y^{*}, z^{*}\right) \in Y \times Z$, although the definition of $\{\theta(x): x \in$ $X\}$ being equi- $L C^{n}$ only requires it for $\left(y^{*}, z^{*}\right) \in \bigcup\{\theta(x): x \in X\}$. 
Since $(V \times H) \cap \theta(x) \neq \varnothing$, we have $(V \times K) \cap \theta(x) \neq \varnothing$, so $V \cap \varphi(x) \prec$ $W \cap \varphi(x)$ by the way $V$ and $K$ were chosen. Hence $\pi_{1} \circ g: S^{m} \rightarrow V \cap \varphi(x)$ extends to a continuous $h: B^{m+1} \rightarrow W \cap \varphi(x)$. Now observe that $\left(W \times N_{\gamma}\left(z^{*}\right)\right) \cap$ $\theta(x) \neq \varnothing$ (since $W \supset V$ and $\left.N_{\gamma}\left(z^{*}\right) \supset H\right)$, so $N_{\gamma}\left(z^{*}\right) \cap \psi(x, y) \neq \varnothing$ for some $y \in W \cap \varphi(x)$, and hence $L \cap \psi\left(x, y^{\prime}\right) \neq \varnothing$ for all $y^{\prime} \in W \cap \varphi(x)$ by the result established three paragraphs back. In particular, $L \cap \psi(x, h(t)) \neq \varnothing$ for all $t \in B^{m+1}$, so we can define $\alpha: B^{m+1} \rightarrow \mathscr{F}(L)$ by

$$
\alpha(t)=L \cap \psi(x, h(t)) .
$$

Note that $\alpha$ is 1.s.c. because $\psi$ is 1.s.c. (by (a)) and because $L$ is open in $Z$. Also each $\alpha(t)$ is a convex subset of $Z$, so each $\alpha(t)$ is $C^{m}$ and $\{\alpha(t): t \in$ $\left.B^{m+1}\right\}$ is equi- $L C^{m}$. Since $L$ is completely metrizable, it follows that $\alpha$ satisfies all the hypotheses of Theorem 1.1 (with $Y$ replaced by $L$ ). Observe next that $\pi_{2} \circ g$ is a selection for $\alpha \mid S^{m}$, for if $t \in S^{m}$ then $g(t) \in(V \times H) \cap \theta(x)$ and hence $\pi_{2}(g(t)) \in H \cap \psi\left(x, \pi_{1}(g(t))\right)=H \cap \psi(x, h(t)) \subset \alpha(t)$. Thus, by Theorem 1.1, $\pi_{2} \circ g$ extends to a selection $k$ for $\alpha$. We can now define $f: B^{m+1} \rightarrow(W \times L) \cap \theta(x)$ by $f(t)=(h(t), k(t))$, and this $f$ is the required extension of $g$.

(g) Define $\xi: A \rightarrow \mathscr{F}(Z)$ by $\xi(x)=\psi(x, g(x))$. It will suffice to show that $\xi$ has a selection $h$, for then the function $g^{\prime}: A \rightarrow Y \times Z$ defined by $g^{\prime}(x)=(g(x), h(x))$ has the required properties. Define $\xi_{i}: A \rightarrow \mathscr{F}(J)$ by $\xi_{i}(x)=\psi_{i}(x, g(x))$. Then $\xi(x)=\prod_{i} \xi_{i}(x)$ (because $\psi(x, y)=$ $\left.\prod_{i} \psi_{i}(x, y)\right)$, so it suffices to show that each $\xi_{i}$ has a selection. Now $\psi_{i}$ is 1. s.c. by $3.1(\mathrm{a})$, and hence so is $\xi_{i}$. Since $\xi_{i}(x)$ is a half open interval $\left(0, u_{i}(x, g(x))\right]$, it follows from [1, Theorem 1.4] that $\xi_{i}$ has a selection.

\section{Proof of Theorem 1.2}

Let $X, Y, \varphi: X \rightarrow 2^{Y}$ and $D \subset X \times Y$ be as in the hypotheses of Theorem 1.1 as modified in Theorem 1.2. Since $D$ is a $G_{\delta}$ in $X \times Y$, we have $D=\bigcap_{i} D_{i}$ with each $D_{i}$ open in $X \times Y$. Since $\varphi$ is equi- $L C^{n}$, we can choose collections $\mathscr{U}_{i}$ of open rectangles in $X \times Y$ as in Lemma 3.2; since $\operatorname{Gr} \varphi \subset D$, we can choose these $\mathscr{U}_{i}$ so that $\bigcup \mathscr{U}_{i} \subset D_{i}$. Let $E \subset X \times Y, Z=(0,1]^{\omega}, \psi: E \rightarrow \mathscr{F}(Z)$ and $\theta: X \rightarrow 2^{Y \times Z}$ be as in Lemma 3.3. Clearly $E \subset D$.

Let us check that $\theta$ satisfies the hypotheses of Theorem 1.1, with $Y$ replaced by $Y \times Z$ and $\varphi$ by $\theta$. First, since $Y$ and $Z$ are completely metrizable, so is $Y \times Z$. Next, $\theta$ is 1.s.c. by 3.3(b). Since $\tilde{\varphi}(x) \in \mathscr{F}(D)$ and thus $\varphi(x) \in$ $\mathscr{F}(D(x))$ for all $x \in X$, and since $\operatorname{Gr} \varphi \subset E \subset D$, we have $\varphi(x) \in \mathscr{F}(E(x))$, and hence $\theta(x) \in \mathscr{F}(Y \times Z)$ by 3.3(c). Finally, $\{\theta(x): x \in X\}$ is equi- $L C^{n}$ by $3.3(\mathrm{f})$.

Now let $A \subset X$ be closed with $\operatorname{dim}_{X} X \backslash A \leq n+1$, and let $g$ be a selection for $\varphi \mid A$. We must show that $g$ can be extended as in the conclusion of 
Theorem 1.1. By 3.3(g), there is a selection $g^{\prime}$ for $\theta \mid A$ such that $\pi_{1} \circ g^{\prime}=g$. By Theorem 1.1 applied to $\theta$ (see the previous paragraph), $g^{\prime}$ extends to a selection $f^{\prime}$ for $\theta \mid U$ for some open $U \supset A$ in $X$; if $\varphi(x)$ is $C^{n}$ for all $x \in X \backslash A$, then so is $\theta(x)$ by 3.3(e), and we can therefore take $U=X$. Letting $f=\pi_{1} \circ f^{\prime}$, we see that $f$ is the required extension of $g$.

\section{Proof of Corollary 1.3}

The following lemma, whose simple verification is omitted, records a useful property of equi- $L C^{n}$ maps. For the definition of $\varphi_{D}$, see the paragraph preceding Corollary 1.3.

Lemma 5.1. Let $\varphi: X \rightarrow \mathscr{P}(Y)$ be equi- $L C^{n}$, and let $D \subset X \times Y$ be open. Then $\varphi_{D}$ is also equi- $L C^{n}{ }^{4}$

Proof of Corollary 1.3. Since $D$ is open in $X \times Y, \varphi_{D}$ is also 1.s.c., and $\varphi_{D}$ is equi- $L C^{n}$ by Lemma 5.1. Clearly $\tilde{\varphi}_{D}(x) \in \mathscr{F}(D)$ for every $x \in X$. We can therefore apply Theorem 1.2, with $A=\varnothing$ and with $\varphi$ replaced by $\varphi_{D}$, to conclude that $\varphi_{D}$ has a selection.

\section{AN APPLiCATION OF COROLLARY 1.3}

In this section, we apply Corollary 1.3 to improve two results in [5]. Our first theorem sharpens [5, Theorem 3.1].

Theorem 6.1. Let $X$ be paracompact, $Y$ a Banach space, and $\varphi: X \rightarrow \mathscr{F}(Y)$ l.s.c. with each $\varphi(x)$ a convex subset of $Y$. Let $E \subset X \times Y$ be closed, ${ }^{5}$ define $X^{\prime}=\{x \in X: \varphi(x) \cap E(x) \neq \varnothing\}$, and suppose that

$$
\operatorname{dim} X<\operatorname{dim} \varphi(x)-\operatorname{dim} \operatorname{conv}(\varphi(x) \cap E(x))
$$

for all $x \in X^{\prime}$. Then $\varphi$ has a selection $f$ such that $f(x) \notin E(x)$ for all $x \in X$.

Proof. Let $D=(X \times Y) \backslash E$. We must prove that $\varphi_{D}$ has a selection, which we do by showing that $\varphi$ and $D$ satisfy the assumptions of Corollary 1.3 with $n$ defined by $\operatorname{dim} X=n+1$.

Clearly $D$ is open in $X \times Y$. Since the intersection of each open ball in $Y$ with each $\varphi(x)$ is convex and thus $C^{n}$, we see that $\{\varphi(x): x \in X\}$ must be equi- $L C^{n}$, and hence $\varphi$ is equi- $L C^{n}$ by Corollary 1.5. Finally, $\varphi_{D}(x)$ is nonempty and $C^{n}$ for all $x \in X$; this is clear if $x \notin X^{\prime}$ (because then $\varphi_{D}(x)=\varphi(x)$ ), and when $x \in X^{\prime}$ it follows from the inequality in the statement of our theorem and from [5, Lemma 2.1] with $K=\varphi(x)$.

${ }^{4}$ By contrast, $\{\varphi(x): x \in X\}$ being equi- $L C^{n}$ does not imply that $\left\{\varphi_{D}(x): x \in X\right\}$ is equi- $L C^{n}$; see Example 7.2.

${ }^{5}$ Theorem 3.1 of [5] makes the stronger (and less simple) assumption that $E$ is the graph of a continuous (with respect to the Hausdorff metric on $\mathscr{F}(Y)$ ) function $\psi: X \rightarrow \mathscr{F}(Y)$. 
The following generalization of Theorem 6.1 sharpens [ 5 , Theorem 3.3], and implies, in particular, that Theorem 6.1 remains true if $E$ is only an $F_{\sigma}$ (rather than closed) in $Y$.

Theorem 6.2. Let $X, Y$ and $\varphi$ be as in Theorem 6.1. Let $E_{i} \subset X \times Y$ $(i=1,2, \ldots)$ be closed, define $X_{i}^{\prime}=\left\{x \in X: \varphi(x) \cap E_{i}(x) \neq \varnothing\right\}$, and suppose that

$$
\operatorname{dim} X<\operatorname{dim} \varphi(x)-\operatorname{dim} \operatorname{conv}\left(\varphi(x) \cap E_{i}(x)\right)
$$

for all $x \in X_{i}^{\prime}$ and all $i$. Then $\varphi$ has a selection $f$ such that $f(x) \notin E_{i}(x)$ for all $x \in X$ and all $i$.

Proof. This result can be derived from Theorem 6.1 just as [5, Theorem 3.3] was derived from [5, Theorem 3.1]. We omit the details.

\section{EXAMPLES}

The following examples are based on the trivial observation that, if $\varphi: X \rightarrow$ $2^{Y}$, if each $\varphi(x)$ is $L C^{n}$ (in the usual sense), and if $X$ is discrete, then $\varphi$ is equi- $L C^{n}$.

Example 7.1. A 1.s.c. $\varphi: X \rightarrow \mathscr{F}(Y)$ such that $\varphi$ is equi- $L C^{n}$ for all $n$ but $\{\varphi(x): x \in X\}$ is not equi- $L C^{0}$.

Proof. Let $X$ be the discrete space of positive integers, let $Y=\mathbf{R}$, and let $\varphi(n)=\{0,1 / n\}$ for all $n \in X$. This $\varphi$ has the required properties.

Our second example is referred to in Footnote 4.

Example 7.2. A 1.s.c. $\varphi: X \rightarrow \mathscr{F}(Y)$ and an open $D \subset X \times Y$ such that $\{\varphi(x): x \in X\}$ is equi- $L C^{n}$ for all $n$ but $\left\{\varphi_{D}(x): x \in X\right\}$ is not equi- $L C^{0}$. Proof. Let $X$ and $Y$ be as in Example 7.1, define $\varphi: X \rightarrow \mathscr{F}(Y)$ by $\varphi(x)=$ $[0,1 / n]$, and let $D=\{(n, y) \in X \times Y, y \neq 1 / 2 n\}$. This $\varphi$ and $D$ have the required properties.

\section{REFERENCES}

1. C. H. Dowker, On countably paracompact spaces, Canad. J. Math. 3 (1951), 219-224.

2. E. Michael, Continuous selections I, Ann. of Math. (2) 63 (1956), 361-382.

3. _ Continuous selections II, Ann. of Math. (2) 64 (1956), 562-580.

4. __ A selection theorem, Proc. Amer. Math. Soc. 17 (1966), 1404-1406.

5. __ Continuous selections avoiding a set, Topology Appl. 28 (1988), 195-213.

Department of Mathematics, University of Washington, Seattle, Washington, 98195 